\title{
Risk factors and results of hemispherotomy reoperations in children
}

\author{
Marcelo Volpon Santos, MD, PhD, 1,2 Thiago Lyrio Teixeira, MD,, Enrico Salomao loriatti, MD,1 \\ Ursula Thome, MD, PhD, ${ }^{2}$ Ana Paula de Andrade Hamad, MD, PhD, ${ }^{2}$ and \\ Helio Rubens Machado, MD, PhD ${ }^{1,2}$ \\ ${ }^{1}$ Division of Pediatric Neurosurgery, Ribeirao Preto Medical School; and ${ }^{2}$ Center for Pediatric Epilepsy Surgery, University \\ Hospital, Ribeirao Preto Medical School, University of São Paulo, Brazil
}

OBJECTIVE The goal of this study was to perform an analysis of a single-center experience with hemispherotomy reoperations for refractory hemispheric pediatric epilepsy due to persistence of seizures after initial surgery. The authors also identify possible anatomical and neurophysiological reasons for hemispherotomy failure, as well as risk factors and surgical options for this subgroup of patients.

METHODS A review was performed of the medical records in 18 consecutive cases in which candidates for redo hemispherotomy were treated between 2003 and 2018 at the authors' epilepsy surgery center. Fourteen patients underwent reoperation due to seizure recurrence and were studied herein, whereas in 3 the initial surgical procedure was stopped because of uncontrollable bleeding, and the remaining patient refused to undergo a reoperation in spite of seizure recurrence and went on to have a vagus nerve stimulation device placed.

RESULTS Among the 14 patients whose seizures recurred and in whom reoperations were done, the etiology of epilepsy consisted of 7 cases with malformations of cortical development ( $50 \%), 5$ cases of Rasmussen encephalitis $(35.8 \%)$, 1 case of porencephaly $(7.1 \%)$, and 1 case of Sturge-Weber syndrome (7.1\%). Eleven patients had radiological evidence of incomplete disconnection. After reoperation, 6 patients were Engel class IA, 1 was Engel II, 5 were Engel III, and 2 were Engel IV, within a mean follow-up of 48.4 months.

CONCLUSIONS Patients with malformations of cortical development have a higher risk of seizure recurrence, and these malformations comprised the main etiology in the reoperation series. Failure of an initial hemispherotomy usually occurs due to incomplete disconnection and needs to be extensively assessed. Outcomes of reoperation are most often favorable, with acceptable complication rates.

https://thejns.org/doi/abs/10.3171/2020.1.FOCUS19944

KEYWORDS epilepsy surgery; hemispherotomy; refractory epilepsy; reoperation

A NATOMICAL hemispherectomy was first reported for epilepsy treatment in 1938 by McKenzie..$^{10,12}$ Since then, the technique has been changed and developed to reach better outcomes with lower complication rates. In 1968 Rasmussen devised the subtotal hemispherectomy technique, as discussed by Bahuleyan et al. and by De Ribaupierre and Delalande. ${ }^{1,6}$ After that, the technique evolved to the parasagittal functional disconnection ${ }^{7}$ and to the periinsular functional hemispherotomy ${ }^{19}$ which are two of the most-used techniques nowadays. ${ }^{1}$

Hemispherotomies are usually indicated for selected patients with seizures refractory to antiepileptic drugs
(AEDs) and with hemispheric epileptic activity-such as patients diagnosed with porencephaly, Rasmussen's encephalitis, Sturge-Weber disease, and hemimegalencephaly. ${ }^{2}$ Good results have frequently been reported, with a mean success rate (seizure freedom or worthwhile improvement) close to $80 \% .^{1}$ However, in some patients who have undergone hemispheric disconnective surgery, disabling seizures might persist; in these situations, redo surgery must be considered. ${ }^{2}$

Given the paucity of studies in the medical literature, the current article aims to discuss possible anatomical and neurophysiological reasons for hemispherotomy failure, 


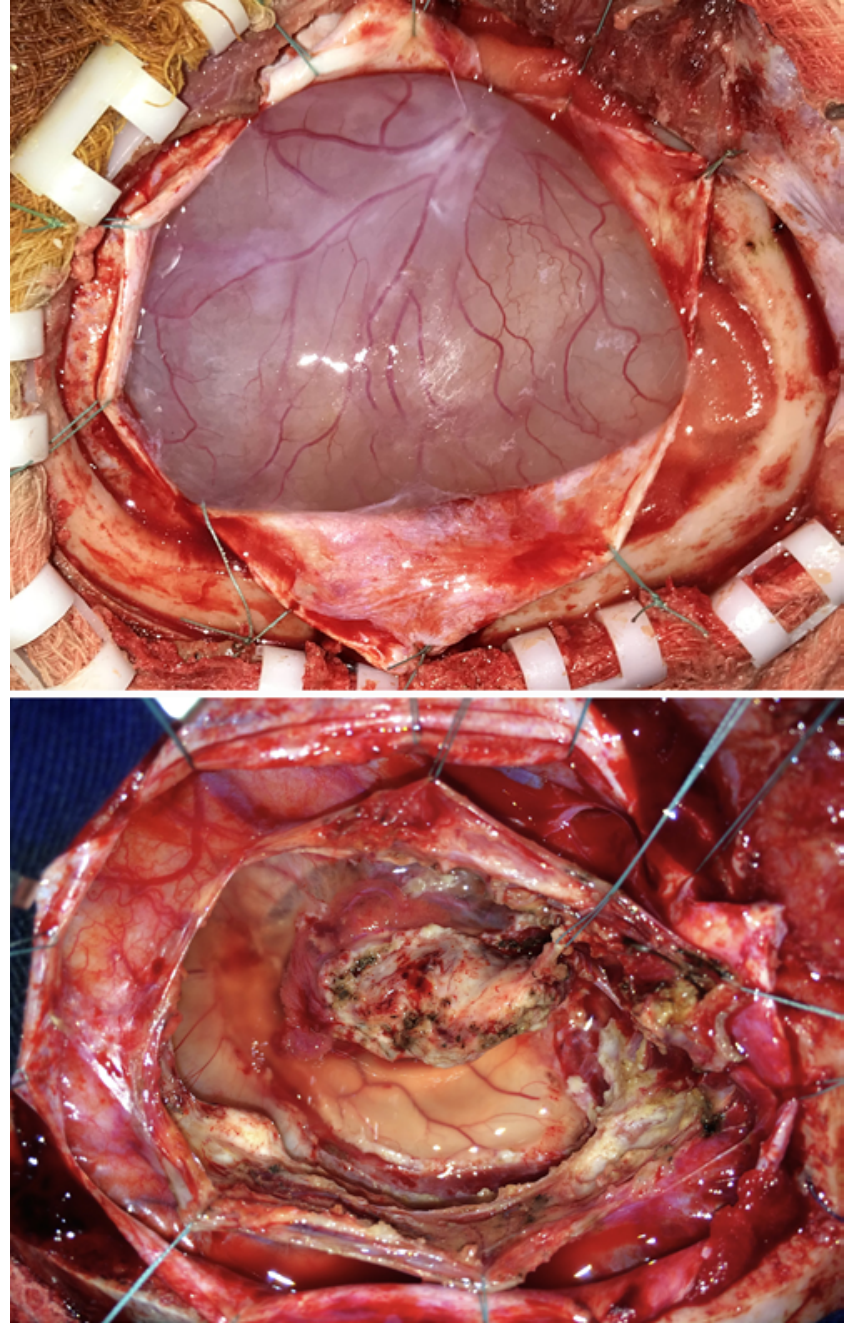

FIG. 1. Upper: Intraoperative photograph depicting the appearance of the cortex during repeated periinsular hemispherotomy. There is usually a large porencephalic space and anatomical structures must be identified with the aid of surgical adjuncts (notably neuronavigation) and can be carefully disclosed as the operation proceeds. Lower: Photographic view after redo disconnection. Completion of corpus callosotomy is warranted after full exposure of the anterior cerebral artery (pericallosal artery), free edge of the tentorium, and tentorial incisura. Wide removal of the temporal lobe and insular cortex is also seen.

and also to identify risk factors and surgical options for this subgroup of patients.

\section{Methods}

We performed a review of the medical records of 18 consecutive patients aged $0-18$ years who were candidates for redo hemispherotomy at our epilepsy surgery center between 2003 and 2018. The following data were collected: age at surgery, etiology of epilepsy, surgical technique, surgical findings at reoperation, MRI features, postoperative seizure status according to Engel's classification, and complications.

All patients underwent routine investigations which included scalp electroencephalography (EEG), video-EEG, high-resolution MRI scans, neuropsychological evalua- tion, nuclear medicine tests, and other convenient examinations when required, pre- and postoperatively. They were further evaluated and discussed in dedicated multidisciplinary team meetings that included pediatric neurologists, neurosurgeons, neuropsychologists, psychiatrists, and social workers, among others, before a final decision regarding surgical therapy was reached.

Redo hemispherotomy was proposed for patients in whom an evident connection between hemispheres was seen on postoperative MRI or in patients with persistent hemispheric epileptic activity rendering refractory seizures.

A periinsular hemispherotomy without temporal lobectomy was the initial surgical strategy in 16 patients (this was also the choice for those whose surgery was interrupted and the one who did not undergo reoperation); the same technique with a temporal lobectomy was performed in one patient, and another underwent a parasagittal hemispherotomy. All operations were carried out by the same senior neurosurgeon, including reoperations. No additional invasive cortical or subcortical monitoring was performed pre- or intraoperatively.

The reoperation technique was chosen according to findings on the postoperative MRI and results of other investigations. No additional invasive cortical or subcortical monitoring was performed during the reoperation procedure. Neuronavigation was used in all of the procedures.

\section{Reoperation Surgical Technique}

Typically, the skin incision and craniotomy are reopened. Durotomy is also the same, and frequently the surgeon starts dissection by creating a surgical plane in the subdural space, releasing the dural-cortical adherences (Fig. 1 upper). Neuronavigation is mandatory. If the preoperative scans had already identified one or more remaining connections (i.e., splenium of corpus callosum or gyrus rectus), they would be approached directly. After completing such disconnections, revision of all other structures that were already disconnected was performed (Fig. 1 lower); a temporal lobectomy was included at the surgeon's discretion, to provide wider exposure and to warrant that brain tissue bridges would not be left behind.

\section{Results}

Overall, 152 patients underwent hemispherotomy at our institution during the abovementioned period; 18 had an indication for reoperation. In 3 of them (all with hemimegalencephaly), the first procedure needed to be interrupted due to uncontrollable bleeding and completed at a later stage. One patient refused reoperation (i.e., redo hemispherotomy) and went on to receive vagus nerve stimulation therapy; therefore, 14 patients actually underwent redo hemispherotomy and were included in the study (Table 1).

Among patients who underwent reoperation, malformations of cortical development (hemimegalencephaly), with 7 cases $(50 \%)$, was the most frequent etiology, followed by 5 cases of Rasmussen encephalitis (35.8\%), 1 case of porencephaly (7.1\%), and 1 case of Sturge-Weber syndrome (7.1\%). Age at first surgery varied from 2 


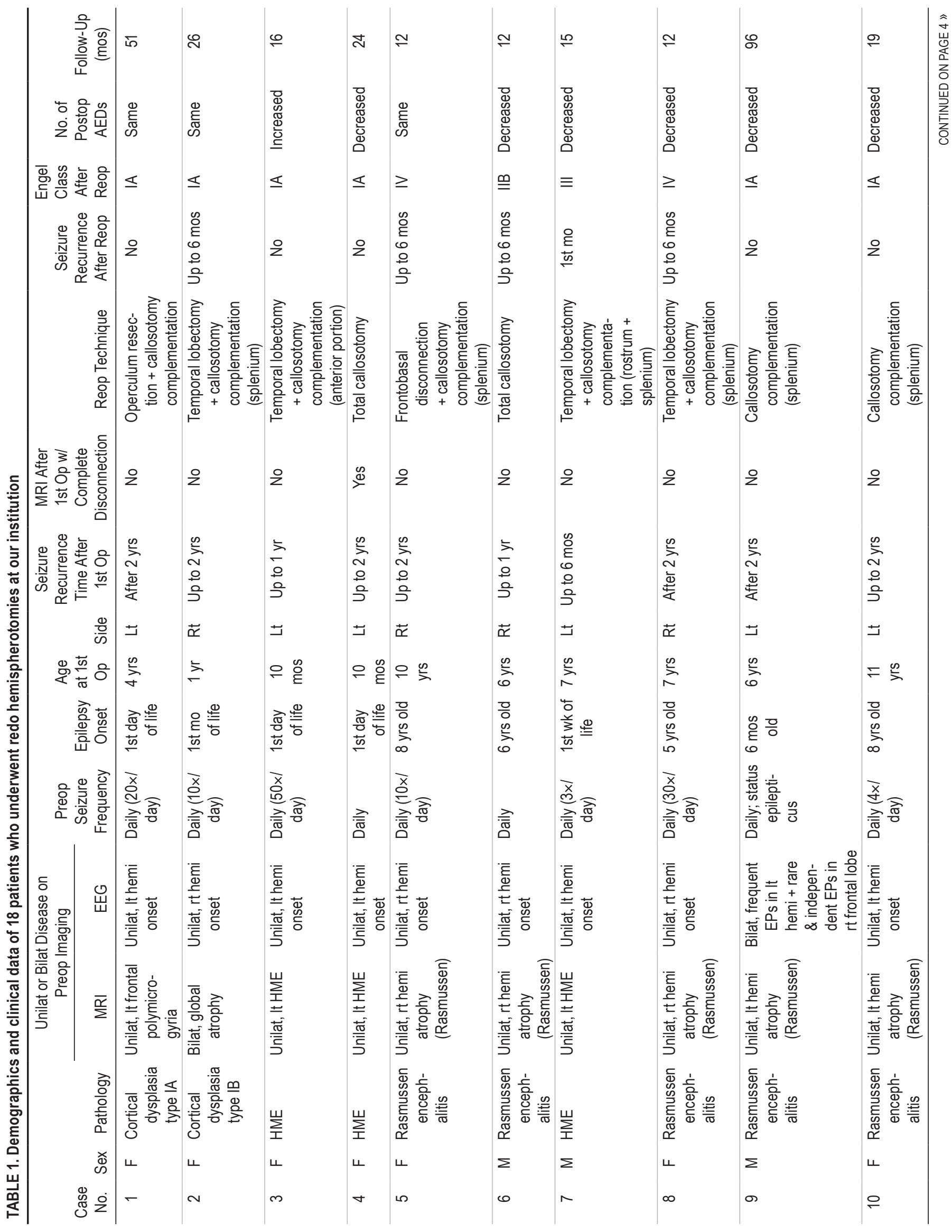




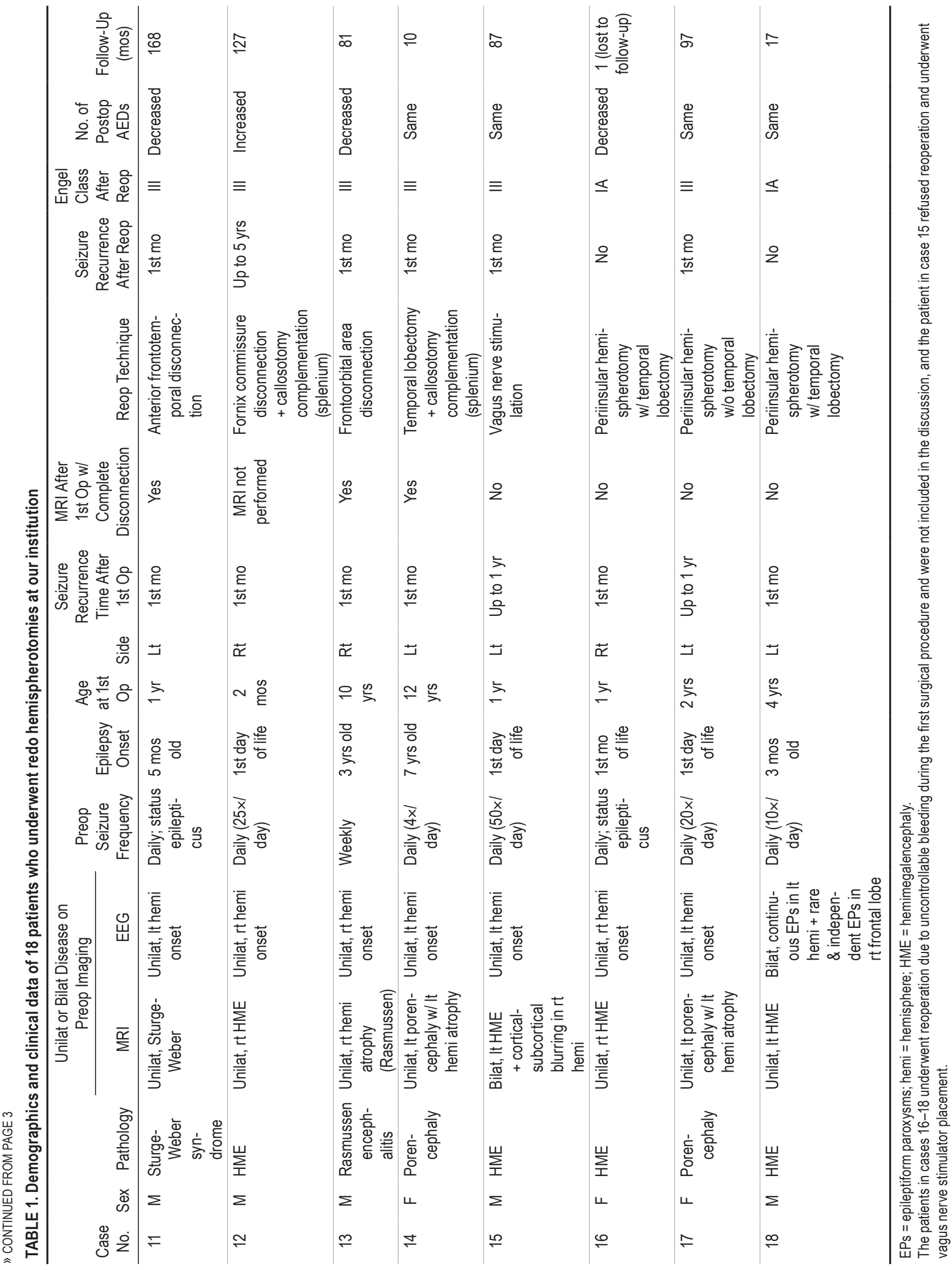


TABLE 2. Summary of the clinical results of published articles reporting on hemispherotomy reoperations

\begin{tabular}{|c|c|c|c|c|}
\hline Variable & Vadera et al., 2012 & Bartoli et al., 2018 & Kiehna et al., 2016 & Present Study \\
\hline No. of patients & 36 & 3 & 8 & 18 \\
\hline Technique & Anatomical hemispherectomy & Not reported & Hemidecortication/periinsular hemispherotomy & Periinsular hemispherotomy \\
\hline Outcome & $\begin{array}{l}64 \% \text { w/ satisfactory seizure control; } \\
19 \% \text { w/ Engel IA; } 36 \% \text { w/ no } \\
\text { improvement }\end{array}$ & $\begin{array}{l}\text { Engel I, 1; Engel II, } \\
1 \text {; Engel III, } 1\end{array}$ & Engel IA, 5; Engel II, 3 & $\begin{array}{l}\text { Engel IA, 8; Engel II, 1; } \\
\text { Engel III, 7; Engel IV, } 2\end{array}$ \\
\hline Follow-up & $7 \mathrm{yrs}$ & 8 yrs & 2 yrs minimum & 4 yrs \\
\hline
\end{tabular}

months to 12 years, with an average age of 6.1 years. At reoperation, age varied from 1 to 18 years, with an average age of 9.1 years.

All 14 patients had refractory epilepsy, with several daily seizures. The onset of epilepsy varied from the first hours of life (5 patients) until 8 years old, with an average age of 1.5 years. Only 1 patient did not have a preoperative focal motor deficit. Three of them had impaired language skills and 13 had various degrees of neurodevelopmental delay at the moment of surgery.

Three patients had no seizure improvement after the first procedure, 2 did not experience significant improvement, and in the other 9 patients, seizures were initially controlled. In 7 patients seizures recurred by the end of the 1st postoperative year, whereas in 7 they recurred after the 1st year of follow-up.

Preoperative MRI scans from 13 patients had just unilateral findings; the remaining patient had bilateral cerebral atrophy. Regarding EEG, although no patient had preoperative bilateral-onset ictal activity before the first surgery, 2 patients had bilateral EEG epileptiform activity $(14.3 \%)$.

Prior to reoperation, 11 patients had radiological evidence of incomplete disconnection, of which 6 were located in the splenium of the corpus callosum, 3 in some other part of the corpus callosum, and 2 had remaining frontobasal connections, which were confirmed intraoperatively. In the 3 cases without previous radiological evidence of incomplete disconnection, the surgical finding was as follows: 1 with incomplete frontobasal disconnection, and 2 with portions of the splenium that remained connected. Five patients also received a temporal lobectomy. Only 4 patients were further studied with tractography after the first failed surgery to identify the remaining connections, all of which identified persistent connecting tracts.

After reoperation, 6 patients achieved Engel class IA, 1 achieved Engel II, 5 achieved Engel III, and 2 had Engel IV, with an average follow-up of 47.7 months. None of this subgroup of 14 patients was lost to follow-up.

Eight patients were able to decrease the number of AEDs used, including 1 patient who was weaned off all AEDs by the time of his last follow-up consultation. Four patients could keep using the same number of AEDs, and 2 patients required an increase in anticonvulsant therapy.

There were 4 complications (28.5\%): 1 surgical wound infection, 1 case of meningitis, and 2 cases of hydrocephalus requiring a shunt. The patient with wound infection required surgical reintervention for debridement and antibiotic treatment, but recovered fully.

\section{Discussion}

Hemispherotomy procedures are indicated for hemispheric diseases that at some point develop into intractable epilepsy, such as diffuse unilateral cortical dysplasia, hemimegalencephaly, Rasmussen encephalitis, SturgeWeber syndrome, and perinatal ischemic events leading to hemispheric injury. ${ }^{2,18}$ Long-term seizure freedom may be achieved in approximately $80 \%$ of patients, ${ }^{2,11,18}$ but $4 \%-$ $9 \%$ of those patients may have no significant improvement after hemispherotomy. ${ }^{2}$ In this scenario, reoperation can improve outcome. ${ }^{14}$ Although seizures may recur even in appropriately selected patients, proper patient selection is still the most important factor in reaching good results. ${ }^{11}$

\section{Reoperation Series and Outcomes}

In the medical literature there are only a few series about redo disconnective procedures after hemispherotomies (Table 2). Even fewer papers discuss surgical indications, risk factors, or outcome of hemispheric reoperations.

In 1996, Peacock reported 5 redo procedures after 27 functional hemispherotomies. ${ }^{15}$ In 2010, Kwan et al. reported a case series of 41 patients, 7 of whom needed second-look surgery for incomplete disconnection, and 3 required a third procedure. ${ }^{9}$

Pinto et al., in 2014, looked separately for two different groups of patients with discrete etiologies who underwent hemispherotomy surgery: one with hemispheric congenital lesions and another with hemispheric acquired lesions. ${ }^{16}$ They noticed that 2 among 22 patients with cortical malformations and none among 14 patients with acquired lesions needed a redo disconnection procedure after an initial functional hemispherectomy, underlining the complexity and difficulty of managing the first group.

Lew et al., in 2013, published a consecutive series of 50 hemispherotomies with 1 reoperation due to incomplete disconnection, achieving Engel IA after that. ${ }^{10}$ These authors also reported that in this same series, the first surgery in 6 patients was done in another hospital and the patients came to their service afterward due to seizure maintenance requiring reoperation. Among these, in the first 3 revisions an anatomical hemispherectomy was performed (Engel outcomes: II, III, and IV), and in the last 3 , disconnections primarily of suspected connected tissue were carried out (Engel class I, I, and II).

Bartoli et al. published in 2018 a more detailed series about 3 cases treated with a second disconnective procedure among 24 primary hemispherotomies. ${ }^{2}$ This series had 1 case of hemimegalencephaly, 1 case of hemispheric 
atrophy, and 1 patient with Rasmussen's disease. MRI findings before the redo procedure showed 2 anterior incomplete callosotomies and 1 posterior callosotomy with frontobasal incomplete disconnection. After reoperation Engel I, II, and III scores were achieved for those patients.

Vadera et al., in 2012, published a series in which they specifically focused on hemispherotomy reoperations. They reported on the characteristics and outcomes of 36 patients who underwent redo anatomical hemispherectomy after initial hemispheric disconnection for epilepsy, and concluded that patients with nonlocalizable or generalized EEG patterns and those with cortical dysplasia as the original cause of seizures may not be ideal candidates for reoperation. ${ }^{18}$ They reported satisfactory seizure control after redo hemispherectomy in $64 \%$ of patients, complete seizure freedom in $19 \%$ of patients, and no improvement in $36 \%$ of patients.

Moosa et al., in 2013, reviewed 186 children who underwent hemispherectomies, finding that bilateral PET abnormalities and acute postoperative seizures were independent predictors of seizure recurrence. ${ }^{13}$ In more than half of those in whom hemispherectomy failed, it did so within the first 6 months after surgery, followed by a slower rate of failures between 1 and 5 years after surgery and a plateau beyond 5 years. For those who needed reoperation after failed hemispherectomy, these authors achieved a $35 \%$ success rate but seizures persisted in $65 \%$, suggesting that the intrinsic epileptogenicity in the other hemisphere could be a major reason for the failure in this situation. These authors also stated that, studying the same patients, early seizure recurrence after hemispherectomy and contralateral hemispheric abnormalities on MRI were major predictors of poor outcome in ambulation, spoken language, and reading.

It is already known that shorter duration of epilepsy prior to surgery is related to better adaptive functioning postoperatively due to the high neuroplasticity of young children. ${ }^{3,13}$ On the other hand, younger age at epilepsy onset is associated with cerebral malformations, which generally carry a poor prognosis, more severe epilepsy, and usually lead to earlier surgery. ${ }^{13,17}$ Therefore, because of this, the exact correlation between age at epilepsy onset and age at first surgery with outcomes needs to be assessed cautiously. Our series could not establish a precise correlation and conclusion for this matter, which would require a greater number of patients.

\section{Etiology}

Congenital malformations are usually the most difficult etiology to achieve complete disconnection., ${ }^{2,16,18}$ Hemimegalencephaly is the most challenging one, due to significant anatomical distortion and a greater propensity to bleeding. A histopathological diagnosis of malformation of cortical development tends to yield worse prognoses

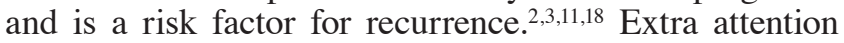
must be given as well to etiologies in which ventricular anatomy is distorted or atypical, ${ }^{10}$ such as Rasmussen's disease or severe hemispheric atrophy.

In our series, hemimegalencephaly was the predominant etiology for incomplete disconnection and redo procedures. Of the overall 152 hemispherotomy cases, 37 had hemimegalencephaly as their primary etiology and 7 (18.9\%) of the 37 had to undergo reoperation. We also had a greater number of Rasmussen encephalitis cases as compared to other literature series-probably because of local factors, because our institution is a reference center for pediatric epilepsy surgery in the country and has a higher number of referrals of patients with Rasmussen's disease than average.

For these so-called difficult etiologies, the use of neuronavigation in the first surgery is even more advisable. Besides, a more extensive opercular and lobar resection might be required for better visualization of the anatomical landmarks, allowing for complete disconnection in some anatomically difficult cases. ${ }^{2}$

\section{Incomplete Disconnection}

Incomplete disconnection might happen regardless of the chosen technique and even in anatomical hemispherectomies..$^{2,5,13}$ Thus, redo disconnective surgery becomes a good option and is often able to achieve satisfactory outcomes. ${ }^{2,8}$

The exact point where disconnection is usually incomplete depends on the technique adopted for hemispheric disconnection and the personal experience of the surgeon. Once this point is identified, more attention must be taken in the initial surgery for this specific step and in the postoperative MRI when looking for reasons of seizure persistence. Tractography is particularly helpful for this assessment. ${ }^{8}$

From an anatomical point of view, the two hemispheres are connected by commissural fibers basically present in the corpus callosum, the anterior commissure, and the hippocampal commissure. The corpus callosum can be divided into anterior genu (connecting the frontal lobes), rostrum, body (connecting the posterior portions of the frontal lobes and the parietal lobes), and splenium (temporal and occipital connections). All connections mentioned above must be interrupted in order to achieve a good outcome. . $^{2,6}$

Connected parts of the anterior corpus callosum and anterior commissure have been reported by some authors as the reason for failure of hemispherotomy. ${ }^{2,48}$ Residual frontobasal white matter connections may represent another possibility. ${ }^{2,18}$ Similarly, among our 14 cases in which reoperation was performed, the splenium of the corpus callosum was by far the main point of the remaining connected tissue, probably because this is the most challenging step during the periinsular hemispherotomy.

Sometimes the point of incomplete disconnection cannot be seen in postoperative MRI scans, especially when tractography is not obtained ${ }^{2,8,18}$ or when the radiology team is not experienced with this kind of procedure. However, when clinical and video-EEG findings suggest unilateral seizure onset, reoperation is still an option and can confirm an incomplete disconnection that the MRI could not pinpoint, as we showed in our results..$^{18}$

Selection of the reoperation technique depends on the findings after the first procedure and the cause of recurrence. Our routine, as described above, is to complete the disconnection when a remaining connection is detected, and to perform a temporal lobectomy only if necessary 
in order to avoid the complications of extensive brain tissue removal. Reopening of the same craniotomy is the first choice when feasible. Some authors prefer to perform an anatomical hemispherectomy for patients who have seizure recurrence after a first hemispheric surgery for epilepsy. ${ }^{18}$ Complete removal of the insular cortex is mandatory when it was not done at the first surgery. ${ }^{2,4}$

\section{Other Causes of Failure}

Bilateral ictal activity and residual insular cortex are reported as other causes of failure of hemispherotomy. ${ }^{2,4,11,13}$ For adequate evaluation, a complete presurgical workup must be performed before a second procedure is considered. ${ }^{2,18}$

Bilateral cerebral activity as documented by EEG bestows a worse prognosis and must be assessed and distinguished from an incomplete disconnection. ${ }^{2,18}$ In our series no ictal activity was initiated in the healthy hemisphere; however, 2 patients had bilateral findings in their preoperative EEG. Curiously, these 2 patients had good outcomes after reoperation (Engel IA and IIA).

Remaining insular cortex can also be the reason for seizure recurrence after hemispherotomy and must be explored in the reoperation procedure. Complete removal includes aspiration of the 3 short and 2 long gyri. ${ }^{2,4}$

\section{Postoperative Complications}

To reduce complications, changes in the surgical technique have evolved from the anatomical hemispherectomy to full functional disconnective surgeries. ${ }^{3}$ Even though complications have indeed decreased, wound infection, bleeding, hydrocephalus, aseptic meningitis, ischemic neurological deficits, and others are still possible in the postoperative period..$^{3,10}$

Intraoperative hemorrhage is a concern, as reported by Basheer et $\mathrm{al}^{3}$ and in the current study, and excessive bleeding occurred in 3 of our original 152 patients in the first procedure, forcing surgery to be stopped and finished in a second time frame. In this situation, hemimegalencephaly is the most frequent etiology.

On the other hand, other issues relating to repeated hemispherotomy are not well described in the literature and do not seem to correlate with the second operation, ${ }^{8}$ nor carry higher risks in comparison with the first procedure. For instance, 2 of our 18 patients required a shunt after reoperation, but in hindsight they already had clinical signs of hydrocephalus prior to the second procedure. Two patients had wound infection and 1 had meningitis-all of them requiring antibiotic treatment but without major complications. Obviously, these are small numbers of patients and do not allow for definitive conclusions.

\section{Conclusions}

Periinsular hemispherotomy is a safe and effective technique used for refractory epilepsy in unilateral hemispheric cerebral disease-with good results. Malformations of cortical development are a risk factor for poor outcome and comprise the main etiology in reoperation series. Incomplete disconnection constitutes the main reason for failed hemispherotomies and needs to be ex- tensively assessed; it usually occurs over the splenium of the corpus callosum, anterior corpus callosum, and frontobasal cortex. Outcomes of reoperation are most often favorable, with acceptable complication rates. Thus, reoperation must be considered in patients with seizure recurrence after hemispherotomy, following an extensive clinical and radiological reevaluation.

\section{References}

1. Bahuleyan B, Robinson S, Nair AR, Sivanandapanicker JL, Cohen AR: Anatomic hemispherectomy: historical perspective. World Neurosurg 80:396-398, 2013

2. Bartoli A, El Hassani Y, Jenny B, Momjian S, Korff CM, Seeck M, et al: What to do in failed hemispherotomy? Our clinical series and review of the literature. Neurosurg Rev 41:125-132, 2018

3. Basheer SN, Connolly MB, Lautzenhiser A, Sherman EM, Hendson G, Steinbok P: Hemispheric surgery in children with refractory epilepsy: seizure outcome, complications, and adaptive function. Epilepsia 48:133-140, 2007

4. Cats EA, Kho KH, Van Nieuwenhuizen O, Van Veelen CW, Gosselaar PH, Van Rijen PC: Seizure freedom after functional hemispherectomy and a possible role for the insular cortex: the Dutch experience. J Neurosurg 107 (4 Suppl):275-280, 2007

5. Cook SW, Nguyen ST, Hu B, Yudovin S, Shields WD, Vinters $\mathrm{HV}$, et al: Cerebral hemispherectomy in pediatric patients with epilepsy: comparison of three techniques by pathological substrate in 115 patients. J Neurosurg 100 (2 Suppl Pediatrics):125-141, 2004

6. De Ribaupierre S, Delalande O: Hemispherotomy and other disconnective techniques. Neurosurg Focus 25(3):E14, 2008

7. Delalande O, Bulteau C, Dellatolas G, Fohlen M, Jalin C, Buret V, et al: Vertical parasagittal hemispherotomy: surgical procedures and clinical long-term outcomes in a population of 83 children. Neurosurgery 60 (2 Suppl 1):ONS19ONS32, 2007

8. Kiehna EN, Widjaja E, Holowka S, Snead OC III, Drake J, Weiss SK, et al: Utility of diffusion tensor imaging studies linked to neuronavigation and other modalities in repeat hemispherotomy for intractable epilepsy. J Neurosurg Pediatr 17:483-490, 2016

9. Kwan A, Ng WH, Otsubo H, Ochi A, Snead OC III, Tamber MS, et al: Hemispherectomy for the control of intractable epilepsy in childhood: comparison of 2 surgical techniques in a single institution. Neurosurgery 67 (2 Suppl Operative):429-436, 2010

10. Lew SM, Matthews AE, Hartman AL, Haranhalli N: Posthemispherectomy hydrocephalus: results of a comprehensive, multiinstitutional review. Epilepsia 54:383-389, 2013

11. Limbrick DD, Narayan P, Powers AK, Ojemann JG, Park TS, Bertrand M, et al: Hemispherotomy: efficacy and analysis of seizure recurrence. J Neurosurg Pediatr 4:323-332, 2009

12. McKenzie KG: The present status of a patient who had the right cerebral hemisphere removed. JAMA 111:168-183, 1938

13. Moosa AN, Jehi L, Marashly A, Cosmo G, Lachhwani D, Wyllie E, et al: Long-term functional outcomes and their predictors after hemispherectomy in 115 children. Epilepsia 54:1771-1779, 2013

14. Muthaffar O, Puka K, Rubinger L, Go C, Snead OC III, Rutka JT, et al: Reoperation after failed resective epilepsy surgery in children. J Neurosurg Pediatr 20:134-140, 2017

15. Peacock WJ, Wehby-Grant MC, Shields WD, Shewmon DA, Chugani HT, Sankar R, et al: Hemispherectomy for intractable seizures in children: a report of 58 cases. Childs Nerv Syst 12:376-384, 1996 
16. Pinto AL, Lohani S, Bergin AM, Bourgeois BF, Black PM, Prabhu SP, et al: Surgery for intractable epilepsy due to unilateral brain disease: a retrospective study comparing hemispherectomy techniques. Pediatr Neurol 51:336-343, 2014

17. Ramantani G, Strobl K, Stathi A, Brandt A, Schubert-Bast $\mathrm{S}$, Wiegand G, et al: Reoperation for refractory epilepsy in childhood: a second chance for selected patients. Neurosurgery 73:695-704, 2013

18. Vadera S, Moosa AN, Jehi L, Gupta A, Kotagal P, Lachhwani $\mathrm{D}$, et al: Reoperative hemispherectomy for intractable epilepsy: a report of 36 patients. Neurosurgery 71:388-393, 2012

19. Villemure JG, Daniel RT: Peri-insular hemispherotomy in paediatric epilepsy. Childs Nerv Syst 22:967-981, 2006

\section{Disclosures}

The authors report no conflict of interest concerning the materials or methods used in this study or the findings specified in this paper.

\section{Author Contributions}

Conception and design: Volpon Santos, Teixeira, Machado. Acquisition of data: Teixeira, Ioriatti, Thome, Hamad. Analysis and interpretation of data: Volpon Santos, Ioriatti, Thome, Hamad. Drafting the article: Volpon Santos, Teixeira. Critically revising the article: Volpon Santos, Machado. Reviewed submitted version of manuscript: Volpon Santos. Approved the final version of the manuscript on behalf of all authors: Volpon Santos.

\section{Supplemental Information}

\section{Previous Presentations}

Portions of this work were presented in abstract form at the 47th Annual Meeting of the International Society for Pediatric Neurosurgery, Birmingham, United Kingdom, on October 22, 2019.

\section{Correspondence}

Marcelo Volpon Santos: Ribeirao Preto Medical School, São Paulo, Brazil.marcelovolpon@usp.br. 